\title{
Adiabatic behavior of gas wells due to natural reservoir fines migration: analytical model and CFD study
}

\author{
S. Senthil ${ }^{1} \cdot$ S. Mahalingam ${ }^{2} \cdot$ S. Ravikumar ${ }^{3}$ (D) $\cdot$ Venkat Pranesh $^{4}$
}

Received: 21 January 2019 / Accepted: 19 April 2019 / Published online: 25 April 2019

(c) The Author(s) 2019

\begin{abstract}
The adiabatic behavior of natural gas production well is frequently neglected in the reservoir formation damage study. The temperature of surrounding formation contributes to the smooth flow of natural gas in the wellbore. The heat transfer from the formation and geothermal gradient plays vital role in the efficient transport of gas in the subsurface formation and wellbore. But, fine particles which are attached to the rock surface migrate and are trapped in the pore throats, and as a result, there is deterioration in the permeability. The detachment of fines occurs due to high reservoir temperature and gas internal energy and temperature. Therefore, this paper conducts theoretical and numerical investigations on the adiabatic behavior of natural gas production well due to in situ reservoir fines migration. Firstly, an analytical model is developed and then, numerical models were constructed using CFD simulation tool to study the adiabatic nature of a gas well. A total of 24 cases were run in simulation, and input temperatures such as $200{ }^{\circ} \mathrm{C}, 250{ }^{\circ} \mathrm{C}, 300{ }^{\circ} \mathrm{C}, 350{ }^{\circ} \mathrm{C}, 400{ }^{\circ} \mathrm{C}$ and $450{ }^{\circ} \mathrm{C}$ were given for modeling. The results revealed that there was no sign of heat transfer from the wellbore to the surroundings. There was a heat circulation only within the wellbore. During the adiabatic state, the pressure in the central zone of the gas well is moderate, but rises linearly on the radial sides.
\end{abstract}

Keywords Gas reservoir · Fines migration $\cdot$ Productivity decline $\cdot$ Adiabatic regime $\cdot$ Temperature $\cdot$ Pressure

\begin{tabular}{ll}
\multicolumn{2}{l}{ List of symbols } \\
RTE & Radiative transfer equation \\
CFD & Computational fluid dynamics \\
$T$ & Temperature \\
$R$ & Gas constant \\
$D$ & Diameter (volume) \\
$Q$ & Heat flow rate \\
$L$ & Total depth of the well
\end{tabular}

Electronic supplementary material The online version of this article (https://doi.org/10.1007/s13202-019-0670-5) contains supplementary material, which is available to authorized users.

S. Ravikumar

ravikumars26@hotmail.com

1 Mechanical Engineering Department, Kamaraj College of Engineering and Technology, Virudhunagar, TN, India

2 Mechanical Engineering Department, Sona College of Technology, Salem, TN, India

3 Mechanical Engineering Department, Jeppiaar Maamallan Engineering College, Sriperumbudur, TN, India

4 Minerals and Inorganic Chemicals Division, Dawn Calorific Exports, Chennai, TN, India
Depth

Enthalpy

Serial number

Radius

$i$ th component

Heat distribution with respect time

Charge per unit mass

Thermal diffusivity

Depth of the reservoir (location from the surface) where the temperature dominates the fine particle detachment

Gas

Entropy

Knudsen diffusion

Klinkenberg effect

Internal energy

Time

Average velocity of gas-oil mixture

Pressure in the porous media

Heat flux in convex surface

Angle

Change in pressure of the porous media

Viscosity of the fluids 


\begin{tabular}{|c|c|}
\hline$\rho$ & Density \\
\hline$\gamma$ & $\begin{array}{l}\text { Continuous heat transfer to porous media } \\
\text { (without any interval) }\end{array}$ \\
\hline$Q_{x}$ & $\begin{array}{l}\text { Instantaneous heat flux flow in porous } \\
\text { media }\end{array}$ \\
\hline$Q_{\mathrm{t}}$ & $\begin{array}{l}\text { Total heat flow in porous media at particu- } \\
\text { lar time }\end{array}$ \\
\hline$\delta_{q}$ & $\begin{array}{l}\text { Kinematic of fluid flow as a function of } \\
\text { time }\end{array}$ \\
\hline$S_{\mathrm{wc}}$ & Connate water saturation \\
\hline$C_{\mathrm{v}}$ & Specific volume \\
\hline $\mathrm{C}_{\mathrm{p}}$ & Specific pressure \\
\hline$K_{\mathrm{g}}$ & Thermal conductivity of gas in reservoir \\
\hline$K_{\mathrm{wc}}$ & $\begin{array}{l}\text { Thermal conductivity of connate water } \\
\text { over rock grain }\end{array}$ \\
\hline$S_{\mathrm{g}}$ & Gas saturation \\
\hline$W_{\mathrm{R}}$ & $\begin{array}{l}\text { Net work transfer to migrate the reservoir } \\
\text { fines in porous media }\end{array}$ \\
\hline$Q_{\mathrm{Lg}}$ & Heat liberation from gas \\
\hline$Q_{\mathrm{Lwc}}$ & Heat liberation from connate water \\
\hline$Q_{\mathrm{wc}}$ & Heat flow in connate water \\
\hline$Q_{\mathrm{hf}}, Q_{\mathrm{cf}}$ & $\begin{array}{l}\text { Gas well inside and outside heat transfer } \\
\text { coefficient }\end{array}$ \\
\hline$W_{\mathrm{R}}$ & $\begin{array}{l}\text { Total work done to displace all fines in } \\
\text { pore surface and for migration in porous } \\
\text { space }\end{array}$ \\
\hline$T_{\mathrm{h} \phi}$ & $\begin{array}{l}\text { Final and highest temperature prevailing in } \\
\text { the porous media }\end{array}$ \\
\hline$T_{\mathrm{i} \phi}$ & $\begin{array}{l}\text { Initial temperature prevailing in the porous } \\
\text { media }\end{array}$ \\
\hline$T_{\mathrm{hf}}$ & Gas flowing temperature in the well \\
\hline$K_{\mathrm{A}}$ & $\begin{array}{l}\text { Thermal conductivity of well inside layer } \\
\text { (wall) }\end{array}$ \\
\hline$K_{\mathrm{B}}$ & Thermal conductivity of the formation \\
\hline$T_{m}, T_{n}$ and $T_{o}$ & $\begin{array}{l}\text { Temperatures in well, casing and cement- } \\
\text { ing, and reservoir formations }\end{array}$ \\
\hline$Q_{1}$ & Heat transfer to porous media \\
\hline$Q_{2}$ & $\begin{array}{l}\text { Internal heat that is already available in the } \\
\text { porous media }\end{array}$ \\
\hline$u_{1}$ & Initial internal energy \\
\hline$u_{2}$ & Final internal energy \\
\hline$\nu_{1}$ & Initial velocity \\
\hline$\nu_{2}$ & Final velocity \\
\hline$h_{1}$ & Initial enthalpy \\
\hline$h_{2}$ & Final enthalpy \\
\hline$s_{1}$ & Initial entropy \\
\hline$s_{2}$ & Final entropy \\
\hline$r_{1}$ & Radius of well \\
\hline$r_{2}$ & Radius of casing \\
\hline$r_{2}$ & Radius of cementing layer \\
\hline$w_{\mathrm{i}}$ & Internal work of mixture \\
\hline
\end{tabular}
$U_{1}$
Initial velocity of gas in porous media (entering the capillary tube from mixing chamber)
$U_{2} \quad$ Final velocity of gas in porous media (leaving the capillary tube to enter another mixing chamber)
$T_{1} \quad$ Initial temperature of pore chamber
$T_{2} \quad$ Final temperature of pore chamber
$T_{\mathrm{h}}-T_{\mathrm{i}} \quad$ Difference in the final and initial reservoir temperature
$\eta_{\phi \mathrm{R}} \quad$ Thermal efficiency of porous media with regard to radiation
$\phi k \quad$ Porosity and permeability
$\frac{\mathrm{d} Q}{\mathrm{~d} t} \quad$ Heat flow rate with respect to temperature change
1 Constant term for additional time, which indicates heat transfer rate for every second

\section{Introduction}

BP Energy Outlook suggests that the global consumption of natural gas is anticipated to rise more rapidly than other fossil fuels, and also the shale gas production rises 5.2\% per year, which is attributed to $60 \%$ of the growth in natural gas production (Dale 2017). But, during production, an inevitable and regrettable occurrence like formation damage possesses a serious threat to the well productivity of natural gas. Gas flow through porous rocks depends on the connected pore space, and in parallel, there exist in situ or natural reservoir fines attached to rock grain composed of clays, usually, kaolinite. Colloidal forces and the imbalance in mechanical equilibrium cause fines to detach from the rock grain to mobilize along with the displacing fluid, thereby plugging the pore space and restricting the permeability which ultimately leads to well impairment and production decline. This mechanism of well impairment is due to fines migration known as reservoir formation damage which harms the natural gas industry. Kaolinite is a common clay which is presented in the porous surface of reservoir rocks, and like colloids, generally, fines have a size of the order $1 \mu \mathrm{m}$ and a net surface charge (Raha et al. 2007). Figure 1 presents a schematic diagram of fine particle under the mechanical forces over a rock surface.

Civan (2010) analyzed the non-isothermal impairment of porous media due to fines migration and deposition and also studied their dispersive transport. He claims the occurrence of this phenomenon was due to variation in temperature and fines mobilization by dispersion and advection. The author developed an analytical model to predict the fines detachment and transport in porous medium. Modeling was performed with and without the consideration of temperature variation and dispersion mechanism. From 


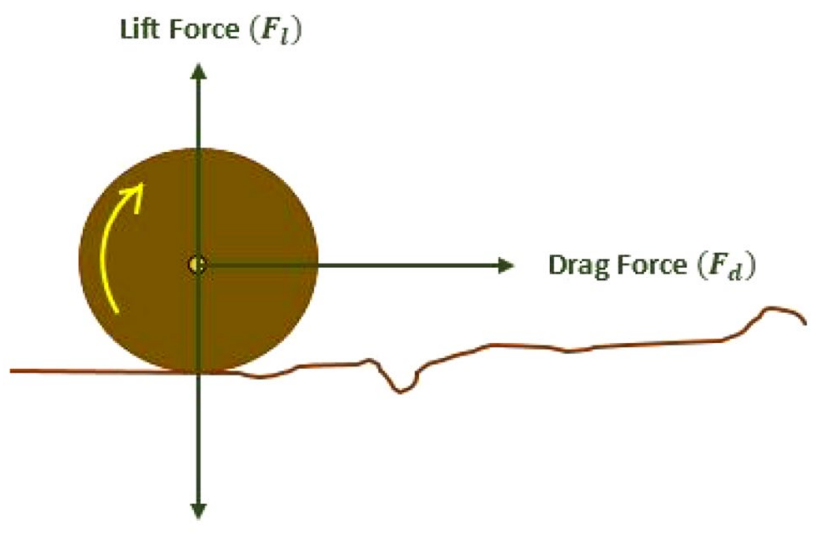

Gravitational and Electrostatic Forces $\left(F_{g}+F_{e}\right)$

Fig. 1 Fine particle which is in the state of equilibrium over the rock surface

the numerical results, it was revealed that a correlation was occurring between varying temperature and fine dispersive mechanics. The difference in porous temperature causes fine particles to disperse which experiences a spreading effect and then impairs the permeability. Overall, Civan research demonstrated that the porous medium temperature variation has a potential effect on fines migration and permeability reduction because it harms the porous matrix thermal deformation, pore throat constriction and the filter coefficient. Additionally, Narasimhan (2013) extensively studied the phenomenon of heat and fluid flow on porous media. He made critical reviews on porous medium heat conduction, forced and natural convection and radiation heat transfer. $\mathrm{He}$ treats the porous medium as porous continuum for modeling purposes, and the energy conservation equations involving volume averaged quantities can be used to estimate the flow and heat transfer models. Also, the radiative heat transfer (RTE) equation determines the radiative intensity propagation, which can be mentioned as below:

$\frac{\partial_{i_{\lambda}}(\Omega)}{\partial \tau_{\lambda}}=I_{\lambda}(\Omega)-i_{\lambda}(\Omega)$

Lin et al. (2013) investigated the two-phase flow and heat transfer in offshore wells. They studied the characteristics of offshore wells operating with electrical submersible pump (ESP). For establishment of heat transfer, the authors analyzed the temperature and pressure distribution in the well and also the flow of oil and gas, which causes the transfer of heat in a radial direction to formations through conduction. They coupled the heat transfer problem with ESP and later performed both analytical and numerical analysis. The authors stated that the petroleum wells exhibit an open thermodynamic system and with this condition the working medium kinematic and thermodynamic parameters which are in open systems and boundary are constant all the time, the fluid flow in the wellbore is considered as steady flow. Hence, changes in kinematic or thermodynamic parameters can comply with the energy equation of steady-state flow of open systems, as given by the first law of thermodynamics (Benjamin 2012):

$\delta_{q}=\mathrm{d} h+\frac{1}{2} \mathrm{~d} v^{2}+g \sin \theta \mathrm{d} z+\delta w_{\mathrm{i}}$

As mentioned in Abstract that in the formation damage study due to fines migration, the adiabatic state or behavior of wells producing natural gas is ignored and neglected. This is the first paper to the best of our knowledge to elucidate the significance of this thermodynamic syndrome and its impact on the gas well productivity. For many researchers, this paper will be unprecedented. For instance, Hagoort (2005) analyzed the wellbore temperatures in gas-producing wells. During his investigation, he found that the well has experienced an adiabatic state during steady-state flow and this is due to the loss in the heat and energy balance between the well and reservoir. However, his research does not deal with formation damage and it should be noted that even without formation damage, under normal conditions, the well has undergone an adiabatic state. Over a period of time, many researchers have not considered this problem deeply and seriously. Even, Zeinijahromi et al. (2012) studied the gas well impairment due to fines migration. In this research, the authors have examined the fines detachment and plugging only at the pore-scale level, which ultimately declined the reservoir rock permeability and fluid flow toward the well. Finally, the gas well experienced a deterioration in the production. Nevertheless, in their research, they did not speak nor explore the thermodynamic (adiabatic) behavior of the wells after reservoir formation damage due to fines migration. Therefore, by comparing the two studies, it can be understood that the former analyzed the adiabatic nature of the well, but did not analyze the formation damage. But, the latter investigated about formation damage due to fines migration and well productivity loss, but did not explore the adiabatic state of the well. This is the major research gap that our paper is trying to explore through analytical and numerical investigations. Even, there was also a claim of adiabatic condition in geothermal wells for about 5 days. Mainly, it was found that the surrounding formation temperature enhances steadily than in the hollow zone of the wellbore (Randolph et al. 2015).

The structure of the paper is described here. The second section describes methodology, which was used in this research, and the third section presents a new analytical model for gas well adiabatic condition prediction. The fourth section critically analyzes the numerical modeling results, and the fifth section concludes the paper. 


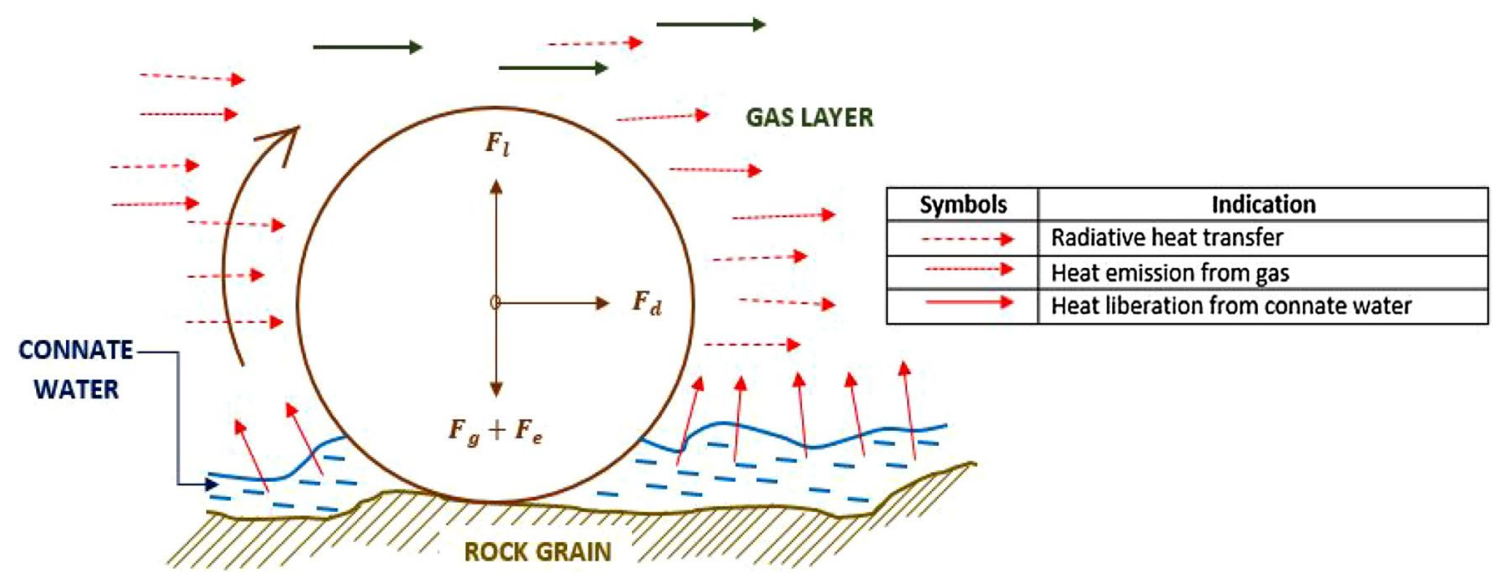

Fig. 2 Attached fine particle under the regime of mechanical forces during gas and heat flow in porous rocks with the presence of connate water. This image is a modified or extended version of Zeinijahromi et al. (2012)

\section{Methodology}

This paper applies computational fluid dynamics (CFD), an integrated fluid mechanics and thermodynamics simulation tool for the numerical modeling of heat transfer, the adiabatic behavior of a gas well. Actually, CFD is rapidly growing as a potential and an alternate simulation tool for reservoir modeling and fluid flow analysis (Cuevas et al. 2010). Traditionally, this tool is widely used by mechanical engineers for flow analysis, but currently CFD ANSYS, FLUENT and CFX are becoming widespread among the reservoir engineering community. For instance, Byrne et al. (2010), used CFD tool for modeling the potential of well inflow in three dimensions. The authors revealed that modeling of conventional well inflow fails to acquire the flow complexity of gas flow into the wells asymmetric around wellbore permeability distribution. Therefore, they understood that a complex numerical model is needed for flow simulation into the horizontal wells with changing levels of formation damage along the well, so that the authors finally employed and recommended the use of computational fluid dynamics simulation tools for solving this concrete specific problem. In our work, the top view of the gas production well and the formation was designed by the CFD ANYSIS simulator. The exchange of heat from the wellbore to formation and vice versa gets declined due to reduction in permeability (as a consequence of fines straining). In this modeling, we assume only the radiation mode of heat transfer and conduction mechanism is neglected. The input values for changing reservoir temperatures are given as $200{ }^{\circ} \mathrm{C}, 250{ }^{\circ} \mathrm{C}, 300^{\circ} \mathrm{C}, 350{ }^{\circ} \mathrm{C}, 400{ }^{\circ} \mathrm{C}$ and $450{ }^{\circ} \mathrm{C}$ with respect to time. The temperature was set to rise in the enclosed two-dimensional cylindrical space. Totally, 24 cases were scheduled for simulation and it ran for about 15 days. Importantly, the entire simulation (that is, in every case) was tested under steady-state flow conditions. Commonly, this type of modeling is needed to be performed under transient state, but initially we tried this flow condition, and our results were irregular and highly exaggerated. Later, we have shifted to investigate this problem under steady-state flow conditions. The required results were successfully obtained, and they will be discussed in the following sections. The main results which were obtained from CFD simulation models are adiabatic profile. Also, plots describing the relationship between temperature, pressure and well diameter are obtained.

\section{Analytical model for the gas well adiabatic behavior due to formation damage}

This section presents a novel analytical model for a natural gas well under an adiabatic state. It should be noted that fines migration will reduce the permeability of a reservoir rock and consequently, there will be a huge decline in the well productivity. Figure 2 is a modified form of Zeinijahromi et al. (2012) layout with the indications of heat liberation from fluids and surroundings. The developed model is for the steadystate gas production with steadily accumulating strained fines that showed linear growth in skin factor against the amount produced in situ fine particles. Also, they have developed a mathematical model for gas production with retained fines accumulation and well productivity. Overall, they have validated the developed analytical model with well productivity of field data which reveals good agreement.

Generally, subsurface porous rocks possess thermodynamic properties such as fluid equilibrium, heat and mass transfer, reservoir fluid phase behavior, internal energy, entropy and exergy. Oil and gas reservoirs together with fines are held within the regime of a thermodynamic force. 
Usually, the pore structure of the porous media in vast majority comprises an interconnected three-dimensional network of capillaries (Dullien 1992). Figure 3 shows the single-system heat transfer model for gas reservoir. The purpose of presenting this figure and model is to displace the fines for achieving the adiabatic state.

As it can be seen from the above figure, the heat is continuously emitted from the hot reservoir that is geothermal gradient to porous reservoir rock (heat engine). Here, porous media are designed and determined to act as a heat engine, which delivers the heat energy to migrate the fluid and fine particle in porous media. Some/excess amount of heat was absorbed over pore walls (heat sink). The extent of the fluid and particle displacement in porous media due to heat transfer is called as thermal efficiency of porous rocks. For the purpose of thermal modeling and analytical investigation, we have coined this new term for porous media. It can be mentioned as follows:

$\eta_{\phi \mathrm{R}}=\frac{W_{\mathrm{R}}}{Q_{1}}$

where $\eta_{\phi \mathrm{R}}=$ Thermal efficiency of porous media with regards to radiation, $W_{\mathrm{R}}=$ network transfer to migrate the reservoir fines in porous media and $Q_{1}=$ heat transfer to porous media.

\section{Gas flow rate under the existence of connate water}

Let us derive the high-velocity equation for the rate of gas flow in the presence of connate water. We know the thermal conductivity equations for gas and connate water.

Let $p=$ pressure in the porous media, $\Delta p=$ change in pressure of the porous media, $U_{1}=$ initial velocity of gas in porous media (entering the capillary tube from mixing chamber), $U_{2}=$ final velocity of gas in porous media (leaving the capillary tube to enter another mixing chamber), $\phi k=$ porosity and permeability and $\mu=$ viscosity of the fluids
By applying the Bernoulli's principle (Bansal 2017), we get the following equation:

$$
\frac{p}{S_{\mathrm{wc}}}+\frac{U_{1}^{2}}{S_{\mathrm{wc}}}+\mu=\frac{\Delta p}{S_{\mathrm{wc}}}+\frac{U_{2}^{2}}{S_{\mathrm{wc}}}+\mu
$$

$$
\left(\frac{p}{S_{\mathrm{wc}}}+\mu\right)-\left(\frac{\Delta p}{S_{\mathrm{wc}}}+\mu\right)=\frac{U_{2}^{2}}{S_{\mathrm{wc}}}-\frac{U_{1}^{2}}{S_{\mathrm{wc}}}
$$

$\left(\frac{p}{S_{\mathrm{wc}}}+\mu\right)-\left(\frac{\Delta p}{S_{\mathrm{wc}}}+\mu\right)=\phi k$

$\phi k=\frac{U_{2}}{S_{\mathrm{wc}}}-\frac{U_{1}}{S_{\mathrm{wc}}}$

$S_{\mathrm{wc}}=U_{2}^{2}-U_{1}^{2}$

$U_{2}^{2}=S_{\mathrm{wc}}+U_{1}^{2}$

$U_{2}=\sqrt{S_{\mathrm{wc}}+U_{1}^{2}}$

$U_{2}=\sqrt{S_{\mathrm{wc}}}+U_{1}$

The above equation can be used to calculate the overall/ final velocity of gas flow rate in porous media under the presence of connate water. On the other hand, if density was taken into consideration, then we can apply the continuity equation in one-dimensional plane to get the following criteria:

$=\rho \times$ Velocity of gas $(\mathrm{d} x) \times$ Connate water saturation $(\mathrm{d} y)$

where

$\rho=$ Density of fluids

$$
=\rho \times U_{2}^{2} \times 2 S_{\mathrm{wc}}
$$

Then, mass of fluid leaving the mixing chamber per second $=\rho U_{2}^{2} 2 S_{\mathrm{wc}}+\frac{\partial}{\partial x}\left(\rho U_{2}^{2} 2 S_{\mathrm{wc}}\right) \mathrm{d} x \mathrm{~d} y$
Fig. 3 Schematic diagram of strained and detached fines due to the influence of geothermal gradient

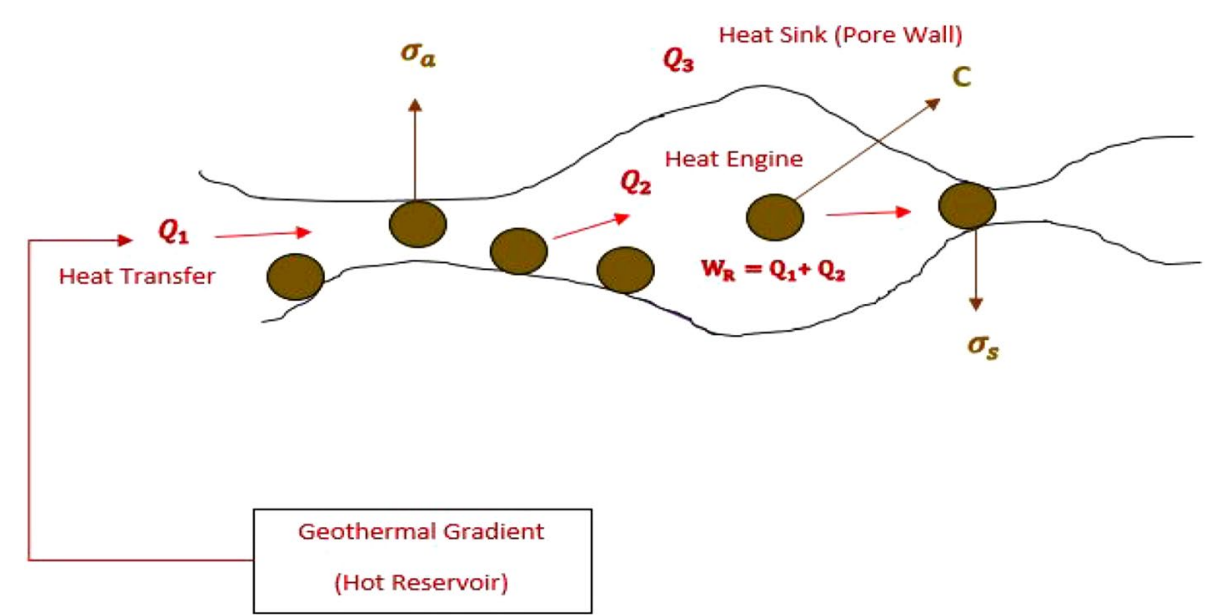


Therefore, gain of the mass in gas flow direction = mass through capillary tube - mass through mixing chamber per second

$=\rho U_{2}^{2} 2 S_{\mathrm{wc}}-\rho U_{2}^{2} 2 S_{\mathrm{wc}}-\frac{\partial}{\partial x}\left(\rho U_{2}^{2} 2 S_{\mathrm{wc}}\right) \mathrm{d} x \mathrm{~d} y$

$=-\frac{\partial}{\partial x}\left(\rho U_{2}^{2} 2 S_{\mathrm{wc}}\right) \mathrm{d} x \mathrm{~d} y$

Similarly, the net gain of the mass in $y$-direction

$=-\frac{\partial}{\partial y}\left(\rho U_{2}^{2} 2 S_{\mathrm{wc}}\right) \mathrm{d} x \mathrm{~d} y$

For steady-state flow, the above should be equated to zero, as shown below:

$\frac{\partial}{\partial x}\left(\rho U_{2}^{2} 2 S_{\mathrm{wc}}\right)+\frac{\partial}{\partial y}\left(\rho U_{2}^{2} 2 S_{\mathrm{wc}}\right)=0$

If the fluid is incompressible, then $\rho$ is constant and the above equation becomes as

$\frac{\partial}{\partial x}\left(U_{2}^{2} 2 S_{\mathrm{wc}}\right)+\frac{\partial}{\partial y}\left(U_{2}^{2} 2 S_{\mathrm{wc}}\right)=0$

In context to thermodynamic modeling, we assume that gas (in reservoir) obeys the following Van der Waal's equation:

$\left(p+\frac{a}{v^{2}}\right)(v-b)=R T$

Now, let us derive the equations for changes in internal energy, enthalpy and entropy of reservoir gas at high-velocity flow. The change in internal energy is given by

$$
\begin{aligned}
& \mathrm{d} u=C_{\mathrm{v}} \mathrm{d} t+\left[T\left(\frac{\partial p}{\partial T}\right)_{\mathrm{v}}-p\right] \mathrm{d} v \\
& \left(\frac{\partial p}{\partial T}\right)_{\mathrm{v}}=\left[\frac{\partial}{\partial T}\left\{\frac{R T}{v-b}-\frac{a}{v^{2}}\right\}\right]_{\mathrm{v}}=\frac{R}{v-b} \\
& \int_{1}^{2} \mathrm{~d} u=c_{\mathrm{v}} \int_{1}^{2} \mathrm{~d} T+\int_{1}^{2}\left[T\left(\frac{\partial p}{\partial T}\right)_{\mathrm{v}}-p\right] \mathrm{d} v \\
& =c_{\mathrm{v}} \int_{1}^{2} \mathrm{~d} T+\int_{1}^{2}\left[T\left(\frac{R}{v-b}\right)-\left\{\frac{R T}{v-b}-\frac{a}{v^{2}}\right\}\right] \mathrm{d} v \\
& =c_{\mathrm{E}} \int_{1}^{2} \mathrm{~d} T+\int_{1}^{2}\left[\frac{R T}{v-b}-\frac{R T}{v-b}+\frac{a}{v^{2}}\right] \mathrm{d} v \\
& =c_{\mathrm{v}} \int_{1}^{2} \mathrm{~d} T+\int_{1}^{2} \frac{a}{v^{2}} \cdot \mathrm{d} v
\end{aligned}
$$

$u_{2}-u_{1}=c_{\mathrm{v}}\left(T_{2}-T_{1}\right)+a\left(\frac{1}{v_{1}}-\frac{1}{v_{2}}\right)$

The change in enthalpy is given by

$$
\begin{aligned}
& \mathrm{d} h=c_{\mathrm{p}} \mathrm{d} T+\left[v-t\left(\frac{\partial v}{\partial T}\right)_{\mathrm{p}}\right] \mathrm{d} p \\
& \left(\frac{\partial h}{\partial p}\right)_{\mathrm{T}}=0+v-T\left(\frac{\partial v}{\partial T}\right)_{\mathrm{p}}
\end{aligned}
$$

Consider $p=f(v, T)$

$\mathrm{d} p=\left(\frac{\partial p}{\partial v}\right)_{\mathrm{T}} \mathrm{d} v+\left(\frac{\partial p}{\partial T}\right)_{\mathrm{V}} \mathrm{d} T$

$(d p)_{\mathrm{T}}=\left(\frac{\partial p}{\partial v}\right)_{\mathrm{T}} \mathrm{d} v+0 \ldots$ as $\quad \mathrm{d} T=0$

From Eq. (13),

$(\mathrm{d} h)_{\mathrm{T}}=\left[v-T\left(\frac{\partial v}{\partial T}\right)_{\mathrm{p}}\right](\mathrm{d} p)_{\mathrm{T}}$

Substituting the value of $(\mathrm{d} p)_{\mathrm{T}}$ in Eq. (14), we get

$$
\begin{aligned}
(\mathrm{d} h)_{\mathrm{T}} & =\left[v-T\left(\frac{\partial v}{\partial T}\right)_{\mathrm{p}}\right]\left(\frac{\partial p}{\partial v}\right)_{\mathrm{T}} \mathrm{d} v \\
& =\left[v\left(\frac{\partial p}{\partial v}\right)_{\mathrm{T}}-T\left(\frac{\partial v}{\partial T}\right)_{\mathrm{p}}\left(\frac{\partial p}{\partial v}\right)_{\mathrm{T}}\right] \mathrm{d} v
\end{aligned}
$$

Use the cyclic relation for $p, v, T$ which is

$\left(\frac{\partial v}{\partial T}\right)_{\mathrm{p}}\left(\frac{\partial T}{\partial p}\right)_{\mathrm{v}}\left(\frac{\partial p}{\partial v}\right)_{\mathrm{T}}=-1$

$\left(\frac{\partial v}{\partial T}\right)_{\mathrm{p}}\left(\frac{\partial p}{\partial v}\right)_{\mathrm{T}}=-\left(\frac{\partial p}{\partial T}\right)_{\mathrm{v}}$

Substituting this value in Eq. (15), we get

$(\mathrm{d} p)_{\mathrm{T}}=\left[v\left(\frac{\partial v}{\partial T}\right)_{\mathrm{p}}+T\left(\frac{\partial p}{\partial T}\right)_{\mathrm{v}}\right] \mathrm{d} v$

For van der Waals equation

$$
\begin{aligned}
\left(\frac{\partial p}{\partial v}\right)_{\mathrm{T}} & =\frac{\partial}{\partial v}\left[\left(\frac{R T}{v-b}\right)-\frac{a}{v^{2}}\right]_{\mathrm{T}} \\
& =-\frac{R T}{(v-b)^{2}}+\frac{2 a}{v^{3}} \\
\left(\frac{\partial p}{\partial T}\right)_{\mathrm{v}} & =\frac{\partial}{\partial T}\left[\left(\frac{R T}{v-b}\right)-\frac{a}{v^{2}}\right]_{\mathrm{V}}=\frac{R}{v-b}
\end{aligned}
$$


Substituting these values in Eq. (16) and Eq. (17) in Eq. (18), we get

$$
\begin{aligned}
& (d h)_{\mathrm{T}}=\left[v\left\{-\frac{R T}{(v-b)^{2}}+\frac{2 a}{v^{3}}\right\}+T\left(\frac{R T}{v-b}\right)\right] \mathrm{d} v \\
& \int_{1}^{2}(d h)_{\mathrm{T}}=-R T \int_{1}^{2} \frac{v}{(v-b)^{2}} \mathrm{~d} v+2 a \int_{1}^{2} \frac{\mathrm{d} v}{v^{2}}+R T \int_{1}^{2} \frac{\mathrm{d} v}{(v-b)}
\end{aligned}
$$$$
K_{\mathrm{wc}}=\frac{\frac{\mathrm{d} Q}{\mathrm{~d} t}}{\left(T_{\mathrm{h}}-T_{\mathrm{i}}\right) \frac{S_{\mathrm{wc}}}{\phi k}}
$$

where $K_{\mathrm{g}}=$ thermal conductivity of gas in reservoir, $K_{\mathrm{wc}}=$ thermal conductivity of connate water over rock grain, $S_{\mathrm{g}}=$ gas saturation, $S_{\mathrm{wc}}=$ connate water saturation, $\frac{\mathrm{d} Q}{\mathrm{~d} t}=$ heat flow rate with respect to temperature change, $\phi k=$ porosity and permeability and $T_{\mathrm{h}}-T_{\mathrm{i}}=$ difference in the final and initial reservoir temperature. The rate of heat flow through a reservoir porous

$$
\begin{aligned}
\left(h_{2}-h_{1}\right)_{\mathrm{T}} & =-R T\left[\log _{e}\left(\frac{v_{2}-b}{v_{1}-b}\right)-b\left\{\frac{1}{v_{2}-b}-\frac{1}{v_{1}-b}\right\}\right]-2 a\left(\frac{1}{v_{2}}-\frac{1}{v_{1}}\right)+R T \log _{e}\left(\frac{v_{2}-b}{v_{1}-b}\right) \\
& =b R T\left[\frac{1}{\left(v_{2}-b\right)}-\frac{1}{\left(v_{1}-b\right)}\right]-2 a\left[\frac{1}{v_{2}}-\frac{1}{v_{1}}\right]
\end{aligned}
$$

The change in entropy is given by:

$\mathrm{d} s=c_{\mathrm{p}} \frac{\mathrm{d} T}{T}+\left(\frac{\partial p}{\partial T}\right)_{\mathrm{v}} \cdot \mathrm{d} v$

For van der Walls equation,

$\left(\frac{\partial p}{\partial T}\right)_{\mathrm{v}}=\frac{R}{v-b}$

as per Eq. (18)

$\mathrm{d} s=c_{\mathrm{v}} \frac{D T}{T}+\frac{R}{v-b} \mathrm{~d} v$

$\int_{1}^{2} \mathrm{~d} s=c_{\mathrm{v}} \int_{1}^{2}\left[\frac{\mathrm{d} T}{T}\right]+R \int_{1}^{2} \frac{\mathrm{d} v}{(v-b)}$

Therefore, we have

$s_{2}-s_{1}=c_{\mathrm{v}} \log _{e}\left[\frac{T_{2}}{T_{1}}\right]+R \log _{e}\left[\frac{v_{2}-b}{v_{1}-b}\right]$.

\section{Heat Transfer model for porous rocks containing gas and connate water}

During heat transfer in porous media holding gas and connate water may experience heat or thermal conductivity, which is mathematically mentioned for gas and water.

For gas,

$$
K_{\mathrm{g}}=\frac{\frac{\mathrm{d} Q}{\mathrm{~d} t}}{\left(T_{\mathrm{h}}-T_{\mathrm{i}}\right) \frac{S_{\mathrm{g}}}{\phi k}}
$$

For connate water, rock under the influence of a particular temperature gradient (typically higher temperature) can be determined by the above two equations. The nomenclature for thermal conductivity is written as capital " $K$," and this can be used to differentiate the permeability "k." This was mentioned to avoid any confusion. Let us derive the heat liberation from in situ porous fluids to mobilize the fines. Heat liberation $\left(Q_{\mathrm{L}}\right)$ from gas and connate water to fine particles (for migration) can be written as:

For gas,

$Q_{\mathrm{Lg}}=K_{\mathrm{g}}-Q_{\mathrm{g}}$

For connate water,

$Q_{\mathrm{Lwc}}=K_{\mathrm{wc}}-Q_{\mathrm{wc}}$

Substituting the values of $K_{\mathrm{g}}$ and $K_{\mathrm{wc}}$ in Eqs. 21 and 22 gives the following:

$Q_{\mathrm{Lg}}=\frac{\frac{\mathrm{d} Q}{\mathrm{~d} t}}{\left(T_{\mathrm{h}}-T_{\mathrm{i}}\right) \frac{S_{\mathrm{g}}}{\phi k}}-Q_{\mathrm{g}}$

$Q_{\mathrm{Lwc}}=\frac{\frac{\mathrm{d} Q}{\mathrm{~d} t}}{\left(T_{\mathrm{h}}-T_{\mathrm{i}}\right) \frac{S_{\mathrm{wc}}}{\phi k}}-Q_{\mathrm{wc}}$

Hence, the total heat liberation $\left(Q_{\mathrm{tot}}\right)$ is given by,

$Q_{\mathrm{tot}}=Q_{\mathrm{Lg}}+Q_{\mathrm{Lwc}}$

$Q_{\mathrm{tot}}=\frac{\frac{\mathrm{d} Q}{\mathrm{~d} t}}{\left(T_{\mathrm{h}}-T_{\mathrm{i}}\right) \frac{S_{\mathrm{g}}}{\phi k}}-Q_{\mathrm{g}}+\left[\frac{\frac{\mathrm{d} Q}{\mathrm{~d} t}}{\left(T_{\mathrm{h}}-T_{\mathrm{i}}\right) \frac{S_{\mathrm{wc}}}{\phi k}}-Q_{\mathrm{wc}}\right]$

$Q_{\mathrm{tot}}=\frac{\frac{\mathrm{d} Q}{\mathrm{~d} t}}{\left(T_{\mathrm{h}}-T_{\mathrm{i}}\right) \frac{S_{\mathrm{g}}}{\phi k}}-Q_{\mathrm{g}}+\frac{\frac{\mathrm{d} Q}{\mathrm{~d} t}}{\left(T_{\mathrm{h}}-T_{\mathrm{i}}\right) \frac{S_{\mathrm{wc}}}{\phi k}}-Q_{\mathrm{wc}}$ 
$Q_{\mathrm{tot}}=\frac{2 \frac{\mathrm{d} Q}{\mathrm{~d} t}}{2\left(T_{\mathrm{h}}-T_{\mathrm{i}}\right) \frac{S_{\mathrm{g}} S_{\mathrm{wc}}}{\phi k}}\left(-Q_{\mathrm{g}}-Q_{\mathrm{wc}}\right)$

$Q_{\mathrm{tot}}=\frac{\frac{\mathrm{d} Q}{\mathrm{~d} t}}{\left(T_{\mathrm{h}}-T_{\mathrm{i}}\right) \frac{S_{\mathrm{g}} S_{\mathrm{wc}}}{\phi k}}\left(-Q_{\mathrm{g}}-Q_{\mathrm{wc}}\right)$

$Q_{\mathrm{tot}}=-\frac{\frac{\mathrm{d} Q}{\mathrm{~d} t}}{\left(T_{\mathrm{h}}-T_{\mathrm{i}}\right) \frac{S_{\mathrm{g}} S_{\mathrm{wc}}}{\phi k}}\left(Q_{\mathrm{g}}+Q_{\mathrm{wc}}\right)$

The above equation gives the overall heat liberated from gas and connate water to fine particles for transport in porous interspace. It can be seen from Fig. 2 that there is heat transfer to porous media through radiation mechanism which is indicated by red arrows. In addition, there are an internal heat generated by the gas flow and other internal heat liberated from the connate water to gas and fine particle, shown in green arrows, which all together contribute to the detachment and migration of fines from the rock surface. Now, let us derive the condition for maximum heat transfer in porous media to mobilize the fine particles. In this condition, the heat source comes from geothermal gradient, which, in turn, will enhance the reservoir temperature and subsequently, impair the electrostatic force and detach the fine particles over the rock surface:

$Q_{x}=\frac{K\left(T_{\mathrm{h}}-T_{\mathrm{i}}\right)}{\sqrt{\pi \alpha \tau}} \exp \left[\frac{\psi^{2}}{4 \alpha \tau}\right]$

where $Q_{x}=$ instantaneous heat flux flow in porous media and $Q_{\mathrm{t}}=$ total heat flow in porous media at particular time

$Q_{\mathrm{t}}=2 K\left(T_{\mathrm{h}}-T_{\mathrm{i}}\right) \sqrt{\frac{\tau}{\pi \alpha}}$

The above equation can be applied for core-scale experimental analysis. $\alpha=$ thermal diffusivity, $\psi=$ depth of the reservoir (location from the surface) where the temperature dominates the fine particles detachment, or we can also consider this symbol as reservoir height/thickness. Here, $\tau=$ heat distribution with respect to time.

To get,

$\frac{d_{Q}}{d_{\tau}}=0 \quad$ or $\quad \frac{d_{Q}}{d_{t}}=0$

$Q_{x}=\frac{K\left(T_{\mathrm{h}}-T_{\mathrm{i}}\right)}{\sqrt{\pi \alpha \tau}} \tau^{-1 / 2} \exp \left[-\frac{\psi^{2}}{4 \alpha \tau}\right]$

$$
\begin{aligned}
& \frac{\mathrm{d}_{Q_{x}}}{\mathrm{~d} t}=\frac{K\left(T_{\mathrm{h}}-T_{\mathrm{i}}\right)}{\sqrt{\pi \alpha \tau}}\left[\left[-\frac{1}{2} t^{-3 / 2} \exp \left[-\frac{\psi^{2}}{4 \alpha \tau}\right]\right]\right. \\
& \left.+t^{-1 / 2} \exp \left[-\frac{\psi^{2}}{4 \alpha \tau}\right]\left(-\frac{\psi^{2}}{4 \alpha} \times-\frac{1}{t^{2}}\right)\right]=0 \\
& =\frac{K\left(T_{\mathrm{h}}-T_{\mathrm{i}}\right)}{\sqrt{\pi \alpha \tau}}\left[\left[-\frac{1}{2} t^{-3 / 2} \exp \left[-\frac{\psi^{2}}{4 \alpha \tau}\right]\right]\right. \\
& \left.+t^{-1 / 2} \exp \left[-\frac{\psi^{2}}{4 \alpha \tau}\right] \frac{\psi^{2}}{4 \alpha t^{2}}\right]=0 \\
& =-\frac{1}{2} t^{-3 / 2} \exp \left[-\frac{\psi^{2}}{4 \alpha \tau}\right]+t^{-1 / 2} \exp \left[-\frac{\psi^{2}}{4 \alpha \tau}\right] \frac{\psi^{2}}{4 \alpha t^{2}}=0 \\
& \frac{1}{2} t^{-3 / 2} \exp \left[-\frac{\psi^{2}}{4 \alpha \tau}\right]=t^{-1 / 2} \exp \left[-\frac{\psi^{2}}{4 \alpha \tau}\right] \frac{\psi^{2}}{4 \alpha t^{2}} \\
& t^{\left(-3 / 2^{+1 / 2+2}\right)}=\frac{2 \psi^{2}}{4 \alpha} \\
& t=\frac{\psi^{2}}{2 \alpha}
\end{aligned}
$$

That is, (since $t=\tau$ )

$\tau=\frac{\psi^{2}}{2 \alpha}$

Equation 30 indicates the condition for maximum heat transfer and with inclusion of a parameter work done in this equation, then:

$\tau=W_{\mathrm{R}}+\frac{\psi^{2}}{2 \alpha}$

We know $W_{\mathrm{R}}=Q_{1}+Q_{2}$

$\tau=\frac{\psi^{2}}{2 \alpha}+\left(Q_{1}+Q_{2}\right)$.

\section{Fines detachment due to heat transfer}

We know very well that $W=$ work done by porous medium to migrate the fine particles and $Q_{1}=$ heat supplied by the source (geothermal gradient). Now, we can derive the fines migration due to heat transfer from Fig. 3 and Eq. 3. By taking this equation, we have

$\eta_{\phi \mathrm{R}}=\frac{W_{\mathrm{R}}}{Q_{1}}$

We know $W_{\mathrm{R}}=Q_{1}+Q_{2}$ where $Q_{2}=$ internal heat that is already available in the porous media.

Substituting the value of $W_{\mathrm{R}}$ in Eq. 3, then

$\eta_{\phi \mathrm{R}}=\frac{Q_{1}+Q_{2}}{Q_{1}}$

Therefore, the above equation can be used to calculate the amount of heat required to displace a fine particle in porous 
media. For continuous increase of heat transfer to porous media, the equation can be written as follows:

$Q_{1}=\frac{\gamma}{\gamma+1} \Delta p\left[\left(\frac{T_{\mathrm{h} \phi}}{T_{\mathrm{i} \phi}}\right)^{\frac{\gamma+1}{\gamma}}+1\right]$

where $Q_{1}=$ total work done to displace all fines in pore surface and for migration in porous space, $\gamma=$ continuous heat transfer to porous media (without any interval), $1=$ constant term for additional time, which indicates heat transfer rate for every second, $\Delta p=$ change in pressure inside porous media, $T_{\mathrm{h} \phi}=$ final and highest temperature prevailing in the porous media and $T_{\mathrm{i} \phi}=$ initial temperature prevailing in the porous media. From this modeling, the detachment and migration of fines are mainly due to heat transfer and high reservoir temperature. Other factors for fines migration were ignored. From Fig. 3, it can be observed that the heat transfer expels and migrates the fine particles in porous interspace and portion of heat is being absorbed and distributed in the pore walls. In some cases, heat will be liberated from the pore walls during water flooding and suspension transport, which will be discussed. In Fig. 3, $C$ indicates the mass concentration of suspended particles and $\sigma_{\mathrm{a}}$ and $\sigma_{\mathrm{s}}$ represent volumetric concentrations of attached and strained fines. Actually, this is Zeinijahromi et al. (2012) model and we have extended and redrew this figure to create a mathematical-based new thermodynamic theory for fines detachment and migration in porous media.

\section{Adiabatic behavior of well after permeability deterioration}

Straining of fine particles in pore throat leads to decline in well productivity, and consequently, the well will exhibit an adiabatic behavior. This does not mean that there would be a decline in the well temperature, and it only indicates that the heat is not transferred from the wellbore to surrounding reservoir rocks due to permeability decline. But, many researchers did not explain about this condition, and they have limited their investigation only till fines plugging and permeability damage. After the gas flow blockage from reservoir to wells (that is after permeability damage), what happens inside the well? This remains a research gap for a long time, and this section gives the possible solution for this behavior. Consider a heat flow to well through porous rocks, and later this heat transfer space will be sealed by natural reservoir fines. Thus, strained fines act as an insulator that is preventing the heat flow toward the well.

Let $T_{\mathrm{hf}}=$ gas flowing temperature in the well, $K_{\mathrm{A}}=$ thermal conductivity of well inside layer (wall), $K_{\mathrm{B}}=$ thermal conductivity of the formation, $T_{m}, T_{n}, T_{o}, r_{1}, r_{2}$ and $r_{3}=$ temperatures and radius of well, casing and cementing and reservoir formations, $L=$ total depth of the well and $Q_{\mathrm{hf}}, Q_{\mathrm{cf}}=$ gas well inside and outside heat transfer coefficient. The rate of heat transfer in the well is given by,

$$
\begin{aligned}
Q & =Q_{\mathrm{hf}} \cdot 2 \pi r_{1} \cdot L\left(T_{\mathrm{hf}}-T_{\mathrm{m}}\right)=\frac{K_{\mathrm{A}} \cdot 2 \pi L\left(T_{m}-T_{n}\right)}{\ln \left(r_{2} / r_{1}\right)} \\
& =\frac{K_{\mathrm{A}} \cdot 2 \pi L\left(T_{n}-T_{o}\right)}{\ln \left(r_{3} / r_{2}\right)}=Q_{\mathrm{hf}} \cdot 2 \pi r_{3} \cdot L\left(T_{o}-T_{\mathrm{hf}}\right)
\end{aligned}
$$

Rearranging the above equations, we get

$T_{\mathrm{hf}}-T_{m}=\frac{Q}{Q_{\mathrm{hf}} r_{1} 2 \pi L}$

$T_{m}-T_{n}=\frac{Q}{\frac{K_{\mathrm{A}} 2 \pi L}{\ln \left(\frac{r_{2}}{r_{1}}\right)}}$

$T_{n}-T_{o}=\frac{Q}{\frac{K_{\mathrm{B}} 2 \pi L}{\ln \left(\frac{r_{3}}{r_{2}}\right)}}$

$T_{o}-T_{\mathrm{hf}}=\frac{Q}{Q_{\mathrm{cf}} r_{3} 2 \pi L}$

Adding (35), (36), (37) and (38), we have

$\frac{Q}{2 \pi L}=\left[\frac{1}{Q_{\mathrm{hf}} r_{1}}+\frac{1}{\frac{K_{\mathrm{A}}}{\ln \left(\frac{r_{2}}{r_{1}}\right)}}+\frac{1}{\frac{K_{\mathrm{B}}}{\ln \left(\frac{r_{3}}{r_{2}}\right)}}+\frac{1}{Q_{\mathrm{cf}} r_{3}}\right]=T_{\mathrm{hf}}$

$Q=\frac{2 \pi L T_{\mathrm{hf}}}{\left[\frac{1}{Q_{\mathrm{hf}} r_{1}}+\frac{1}{\frac{K_{\mathrm{A}}}{\ln \left(\frac{r_{2}}{r_{1}}\right)}}+\frac{1}{\frac{K_{\mathrm{B}}}{\ln \left(\frac{r_{3}}{r_{2}}\right)}}+\frac{1}{Q_{\mathrm{cf}} r_{3}}\right]}$

Therefore,

$Q=\frac{2 \pi L T_{\mathrm{hf}}}{\left[\frac{1}{Q_{\mathrm{hf}} r_{1}}+\frac{1}{\frac{\ln \left(\frac{r_{2}}{r_{1}}\right)}{K_{\mathrm{A}}}}+\frac{1}{\frac{\ln \left(\frac{r_{3}}{r_{2}}\right)}{K_{\mathrm{B}}}}+\frac{1}{\frac{Q_{\mathrm{cf}}}{r_{3}}}\right]}$

If we consider $n$ as rise in well temperature under adiabatic condition, then

$Q=\frac{2 \pi n T_{\mathrm{hf}}}{\left[\frac{1}{Q_{\mathrm{hf}} r_{1}}+\sum_{n=1}^{n=n} \ln \left\{r_{(n+1)} / r_{n}\right\}+\frac{1}{Q_{\mathrm{cf}} r_{(n+1)}}\right]}$ 
Fig. 4 Schematic diagram of adiabatic behavior of gas well due to migration and straining of natural reservoir fines

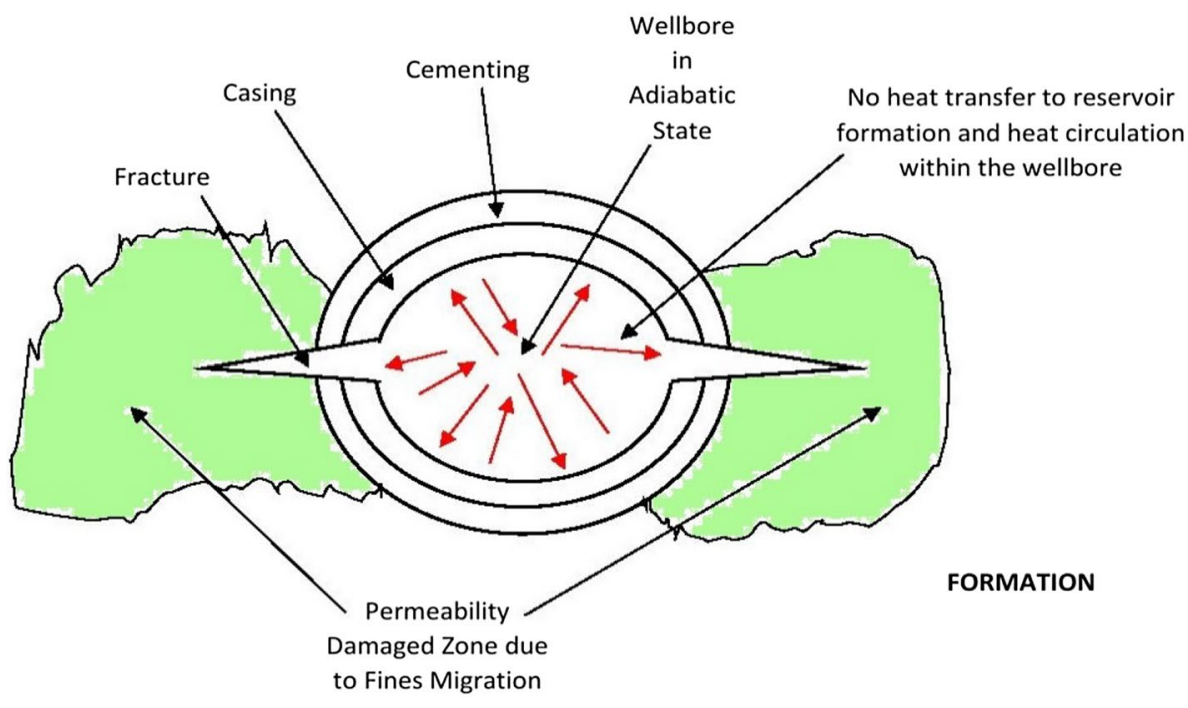

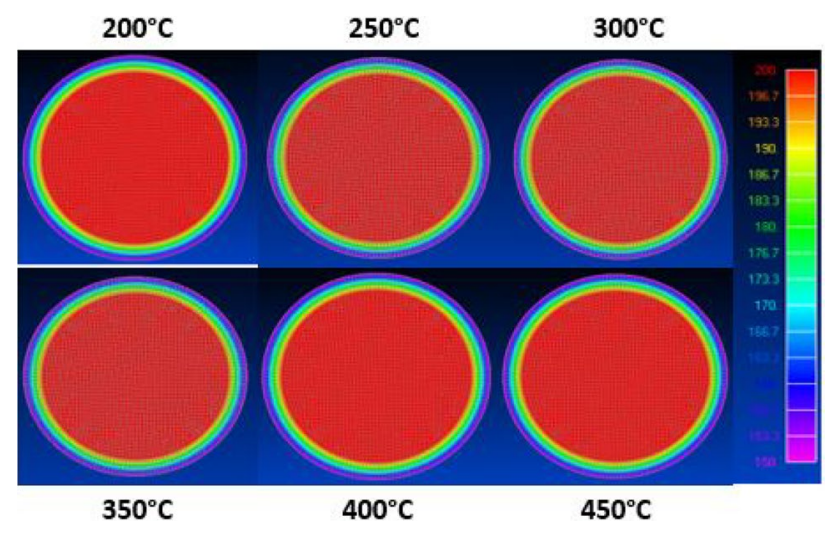

Fig. 5 Well temperature variation at different steady-state thermal regimes (for the view of figures with labels and coordinates, a reader is advised to see Online Appendix A1)

For an adiabatic state, if the well and reservoir formation heat transfer coefficients are not considered, then the above equation can be modified as

$Q=\frac{2 \pi L T_{\mathrm{hf}}}{\left[\sum_{n=1}^{n=n} \ln \left\{r_{(n+1)} / r_{n}\right\}\right]}$

Hence, heat transfer modeling is successfully applied to gas flow permeability decline and well impairment due to fines migration. Figure 4 shows the schematic diagram of gas well adiabatic behavior due to migration and straining of in situ reservoir fines. In this model, fines act as an insulator, and then the well will experience a state of adiabatic condition, which means there will not be any transfer of heat from the wellbore to reservoir formation. Only phenomenon is the heat will be circulating within the wellbore, and the thermal conductivity mechanism of heat transfer is not considered.
Consequently, well may experience higher (abnormal) pressure than the normal gas flowing pressure.

\section{Results and discussions}

This section critically analyzes the numerical modeling results of an adiabatic gas well. These results were acquired from the CFD-ANSYS simulation tool.

\section{Temperature formation in well under adiabatic state}

Figure 5 presents well temperature variation at different steady-state thermal regime. After the fines capture (also known as straining) in the pore throat, there will be a decline in the gas flow since the rock permeability is damaged. Before this occurrence, some volume of the gases already have entered the wellbore and heat transfer exchange rates between well and formations undergo an exponential drop. In this case, we assume there is no means of heat transfer through conduction or convection mechanisms, but only radiation. Consequently, the wellbore will experience a thermodynamic state of adiabatic compression and this may lead to abnormal pressures in the well that in turn causes an explosion, structural failure and similar well control problems. So at this time, the well is shut down and as a result the heat and gas will be circulating in the wellbore.

It can be seen from Fig. 5 that at almost all temperature regimes an adiabatic state is displayed. In the steady-state thermal regimes of $200{ }^{\circ} \mathrm{C}, 250{ }^{\circ} \mathrm{C}, 300{ }^{\circ} \mathrm{C}, 350{ }^{\circ} \mathrm{C}, 400{ }^{\circ} \mathrm{C}$ and $450^{\circ} \mathrm{C}$, the temperature inside the well is extremely high and with no sign of variation or drop in the temperature. It can be observed that the temperature rises linearly and after some time it gets saturated. Not only geothermal gradient, 


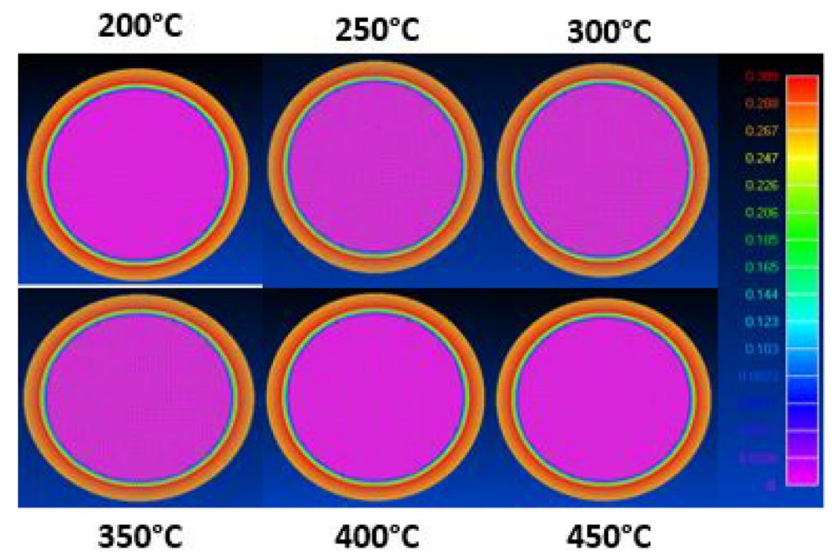

Fig. 6 Heat flux across the well at different steady-state thermal regimes (for the view of figures with labels and coordinates, a reader is advised to see Online Appendix A1)

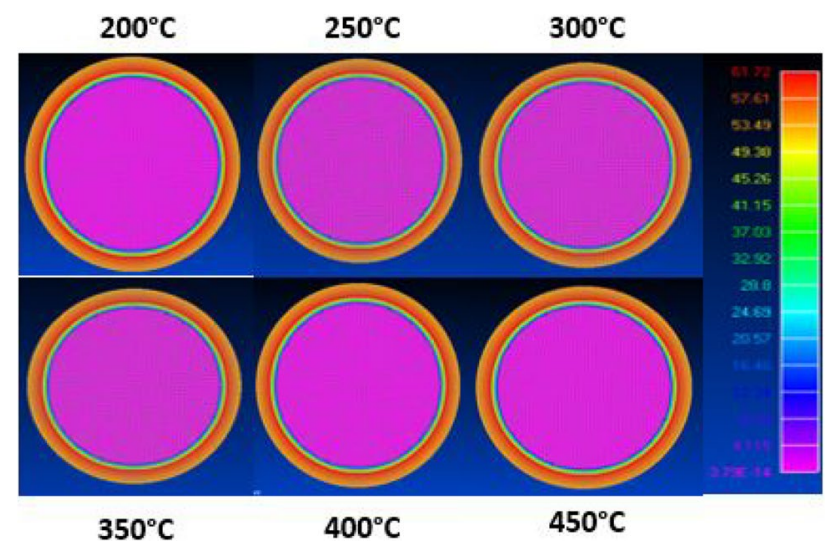

Fig. 7 Temperature gradient across the well at different steady-state thermal regimes (for the view of figures with labels and coordinates, a reader is advised to see Online Appendix A1)

but the gas in the wellbore also contributed to the well temperature elevation. There is no heat loss inside the well when it is in the adiabatic state, but there is a loss in heat balance between the perforation region of wellbore and formation. Conversely, heat flux across the well at each steady-state thermal regime is shown in Fig. 6. In this point, the wellbore under adiabatic state has undergone a transient flow. As a result, there was a sudden drop in temperature. This drop is only in the marginal scale, but not so severe, and heat loss is also may be due to the disturbances in the heat balance between these two systems.

In the viewpoint of Ramey (1962), heat loss in the heat and energy balance between the two thermodynamic systems can be attributed to steady-state heat flow near the well and in the transient state is governed by the conduction mechanism of heat propagation in the reservoir formation. Moreover, the wellbore and formation heat transport

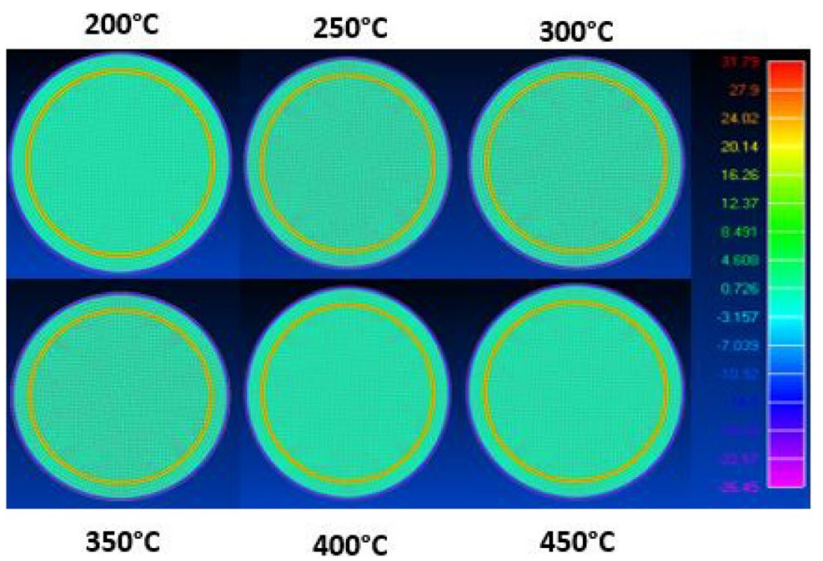

Fig. 8 Thermally constrained load at different steady-state thermal regimes (for the view of figures with labels and coordinates, a reader is advised to see Online Appendix A1)

occurs chiefly in the perpendicular direction of the wellbore. Actually, in our modeling we assume that there is no heat transfer via conduction. The heat balance is affected in the steady-state flow, and this is in good agreement with his arguments. Additionally, Hagoort (2005) indicates that the practical importance of this scenario is that the adiabatic temperature drop suggests that the heat propagation in the wellbore increases the temperature drop. But, our numerical models were slightly offset to the Hagoort's findings. Since our results exposed that there is no heavy drop in the well temperature, there are only an oscillation and marginal saturation. This well adiabatic expansion behavior was even observed for the temperature gradient at all steady-state thermal regimes. Most importantly, it should be taken into an account that in all cases there is no transfer of heat between the wellbore and formation face. It can be seen from Fig. 7 that the formation face temperature (orange) is higher than the inside well temperature (indicated in pink).

Figure 8 shows the thermally constrained load at different steady-state thermal regimes. At all thermodynamic regimes, the thermally constrained load is constant, but only the intensity varies. The adiabatic well generates in situ stress and that in turn will create tensile forces (Zhang et al. 2015). These forces will possess from either side of the systems, wellbore and formation. During this process, there will be an imbalance in the mechanical forces and subsequently, the wellbore and the formation face temperature plummets rapidly. All six images in Fig. 8 showed moderate temperature distribution. It can also be implied that the gas and heat circulation in the wellbore generates stresses, which ultimately leads to tensile stress in all radial sides that is from the wellbore to formation face. Additionally, the same stress is exerted from the internal reservoir zones, thus making the temperature profile in the wellbore to neutralize. This kind of phenomenon is even achieved at the extreme 
Fig. 9 Variation in temperature across the internal diameter of the well
Fig. 10 Variation in pressure across the well internal diameter
Temperature Across Well Diameter

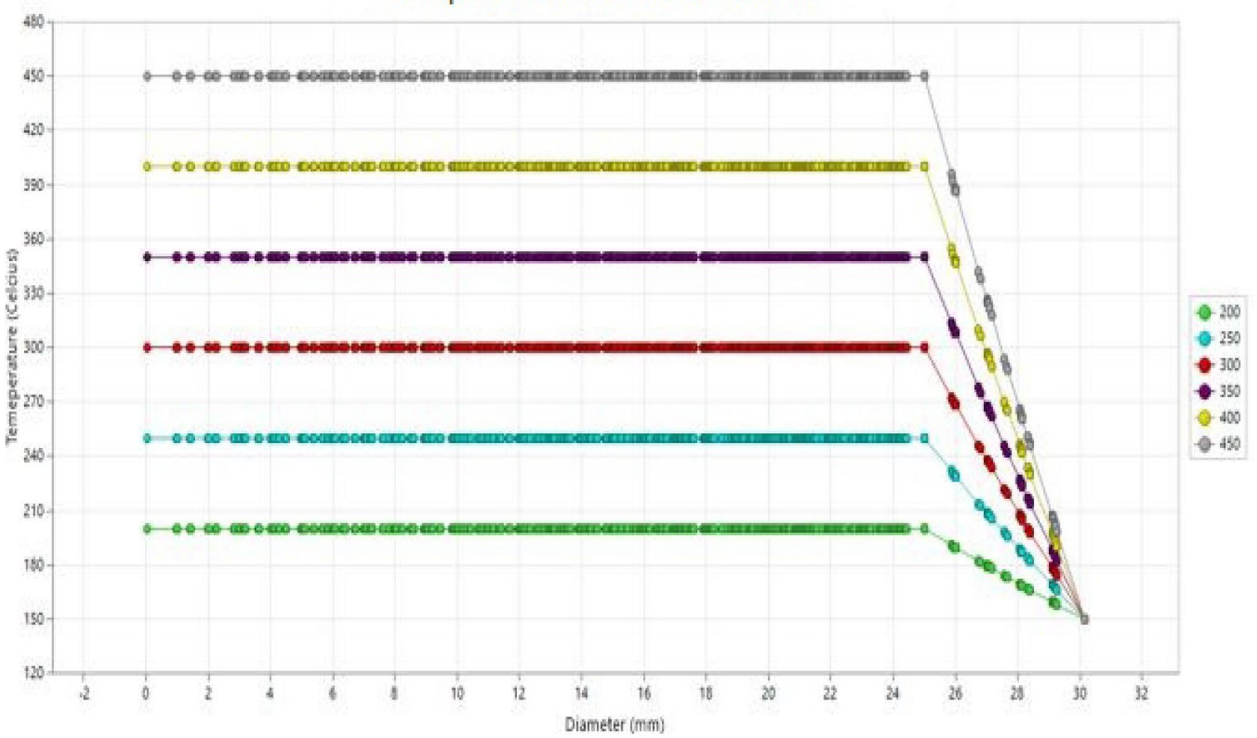

Pressure Across Well Diameter

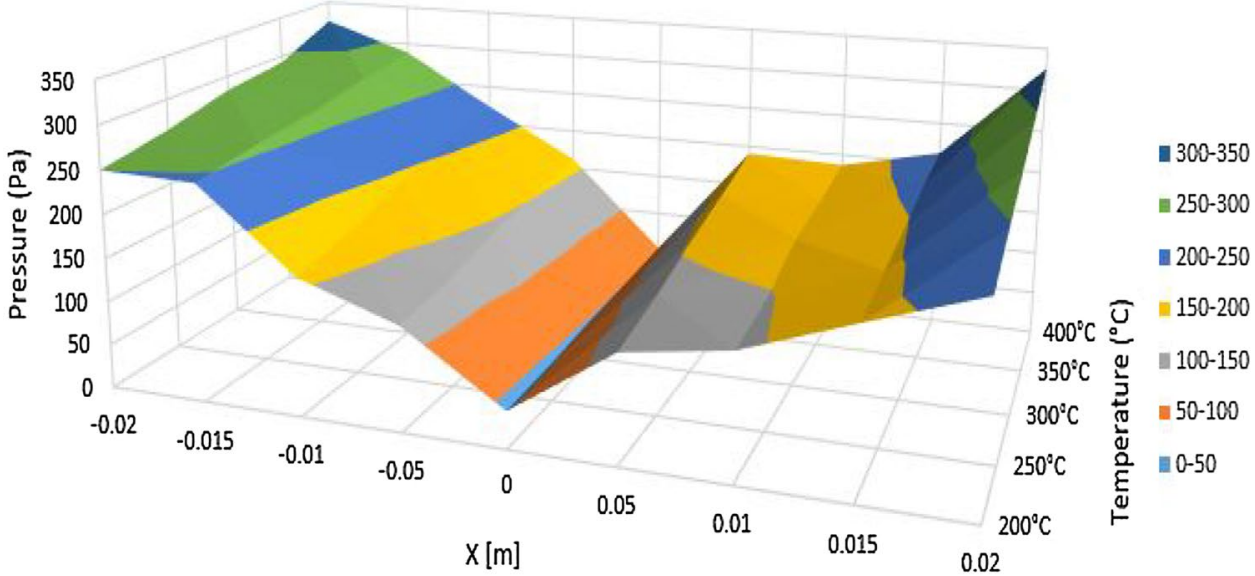

elevated reservoir temperatures of $300{ }^{\circ} \mathrm{C}, 350{ }^{\circ} \mathrm{C}, 400{ }^{\circ} \mathrm{C}$ and $450{ }^{\circ} \mathrm{C}$. Henceforth, this paper successfully elucidated and exhibited the adiabatic behavior of a gas well by numerical modeling.

\section{Impact of well internal diameter on temperature and pressure}

This section presents the temperature and pressure distribution across the gas well under adiabatic state with particular emphasis on the wellbore internal diameter. Figure 9 indicates the temperature variation across the well.

It can be seen from this figure that the temperature rises gradually on the radial ride of the wellbore and at a particular stage it becomes constant without any small variations. It was tested for the wellbore temperatures such as $200{ }^{\circ} \mathrm{C}$,
$250{ }^{\circ} \mathrm{C}, 300{ }^{\circ} \mathrm{C}, 350{ }^{\circ} \mathrm{C}, 400{ }^{\circ} \mathrm{C}$ and $450{ }^{\circ} \mathrm{C}$. But, all regimes exposed same behaviors. Initially, the wellbore axis temperature is close to $150{ }^{\circ} \mathrm{C}$ and then gradually rises to an extreme saturation level. Typically, this type of behaviors is observed in gas wells flowing under the state of non-Darcy flow that is in turbulent flow. In this scenario, occurrence of turbulence is almost impossible due to the adiabatic nature of the well. Also, the flow rate was declining nor stopped due to fines plugging in the pore throat. The moderate temperature at the axis of the wellbore is mainly due to the radial flow of the gas from the surrounding formation to the wellbore. During that time, there will be an impact on the energy and heat balance. As a result, the central zone of the well may undergo a thermal oscillation or even a slight drop in the temperature. Figure 10 shows the pressure change with regard to well internal diameter. It can be observed 


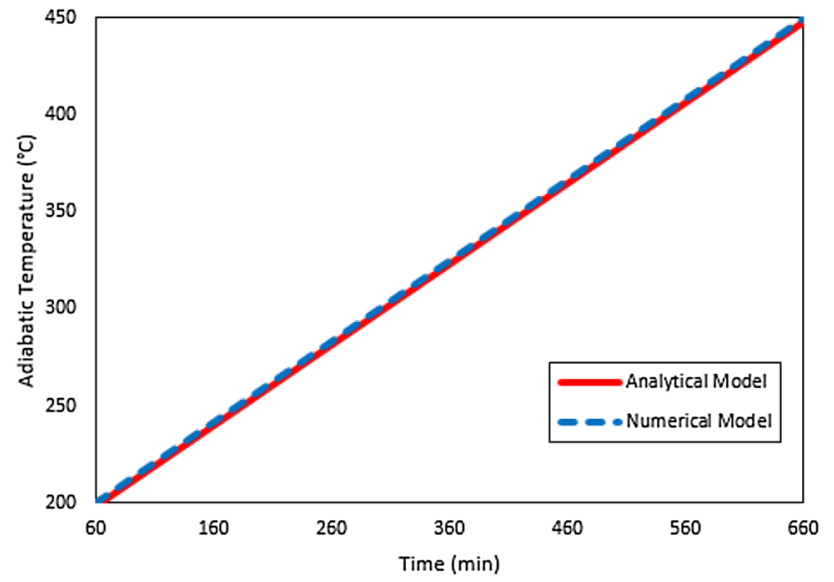

Fig. 11 Analytical and numerical model correlations for an adiabatic gas well

from this figure that non-uniformly distributed in the radial sides of the wellbore. A similar observation was noted as that of temperature profile. It is well known that with context to thermodynamics the temperature is directly proportional to the pressure. If the temperature rises in a linear direction, contemporarily, the pressure climbs in that direction. It can be observed from Fig. 11 that on the left radial side the pressure distribution is moderately saturated and other side (right) this is not recorded. Therefore, it can be inferred that on the zones near to casing wall the pressure may prevail constant nor appear high. This was evident from the previous section, where the temperature distribution is in between medium to high in near regions of the well wall.

It can be seen from Fig. 11 that there is a close correlation between numerical and analytical models. The correlation was plotted between the simulation running time and the adiabatic temperature of a gas wellbore. The major factors such as temperature (both wellbore and formation), pressure, mass flow rate were taken for the calculation. The calculations and models revealed that there are no significant differences between these models. Therefore, this model is reliable for field- and laboratory-based investigations on the adiabatic conditions in the wells producing natural gas.

\section{Conclusions}

This paper successfully demonstrated the adiabatic behavior of a natural gas well due to in situ reservoir fines migration. Based on the analytical and numerical modeling, the following conclusions can be drawn:

1. We have presented a new analytical model for fines migration and well adiabatic state prediction. In analytical modeling itself, it was observed that the reservoir temperature is a dominating factor in the detachment and migration of fines in the porous media. Higher temperature due to geothermal gradient decreases the electrostatic attraction of the fines over the rock surface.

2. An adiabatic state of a gas well is visualized in the numerical modeling results. It clearly showed that there is no radiative heat transfer from the wellbore to formation. Heat is being circulated within the wellbore, and as a result, the temperature and pressure rapidly increase. Even at extreme rising temperatures in the wellbore, there was no sign of heat transfer to the surroundings. Natural gas internal energy and thermodynamics contribute to the proliferation of temperature and pressure in the wellbore during adiabatic state.

3. Well diameter plays a crucial role in the determination of temperature and pressure of the well. The temperature and pressure of the gas well rise radially, and its axial direction is moderate. Overall, the analytical and numerical models revealed a good agreement. Therefore, this paper presented a reliable thermodynamic model for fines transport in porous media and gas well adiabatic prevalence investigations. In our future work, we shall take efforts to correlate this theoretical cum numerical modeling work with well temperature measurements.

Acknowledgements VP thanks Mr. Sakthivel Murugan, assistant professor of mechanical engineering at KCET, Virudhunagar, India, for the fruitful discussions on the computational fluid dynamics concepts and models. Additionally, we thank the anonymous reviewer for invaluable comments to improve the quality of this paper.

Open Access This article is distributed under the terms of the Creative Commons Attribution 4.0 International License (http://creativeco mmons.org/licenses/by/4.0/), which permits unrestricted use, distribution, and reproduction in any medium, provided you give appropriate credit to the original author(s) and the source, provide a link to the Creative Commons license, and indicate if changes were made.

\section{Appendix 1: Negligence of heat conduction}

In this research work, the heat conduction at the interface between the well and the rock is neglected for numerical modeling. It can be observed from the simulation results that there is no heat transfer between steel wellbore casing and reservoir rock formation, and vice versa. When there is no heat transfer between two systems, it means that there is no existence of heat conduction. For example, Hagoort (2005) studied the adiabatic and non-adiabatic temperature profiles of wellbore producing natural gas. He stated that the differences in the heat, mass, and energy balance between the wellbore and reservoir make the wellbore behave adiabatically. Moreover, Mu and Mu (2013) stated that the oil and gas reservoir acts as a layer of thermal insulation. So

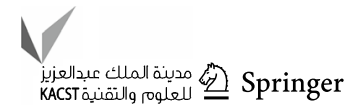


the heat from the geothermal gradient that is from very deep subsurface rocks near mantle cannot pass through this thermal insulation layer and cannot reach the surface or even shallow depth. The implication from their theory is that the hydrocarbon reservoir even sometimes behaves adiabatically, and obviously, there is no option for heat flow in subsurface porous media. Therefore, most researchers are not considering and exploring the heat and mass transfer during reservoir engineering and well testing calculations.

\section{Appendix 2: Well adiabatic behavior at extreme high temperatures}

It is perspicacious for a reader to claim that the natural gas reservoir may not reach extreme temperatures like $300^{\circ} \mathrm{C}$, $350{ }^{\circ} \mathrm{C}, 400^{\circ} \mathrm{C}$ and $450{ }^{\circ} \mathrm{C}$. We agree with the reader that it is a very rare occurrence of a natural gas reservoir to reach a temperature between 300 to $450{ }^{\circ} \mathrm{C}$ regardless of conventional or unconventional formations. We could have stopped our research till $250{ }^{\circ} \mathrm{C}$, but continued to $450{ }^{\circ} \mathrm{C}$. This is purely for the purpose of curiosity and theoretical perspective. It can be observed from the simulation results that at $200{ }^{\circ} \mathrm{C}$ and $250{ }^{\circ} \mathrm{C}$ there was no heat transfer or exchange between wellbore and reservoir. Furthermore, we want to find the feasibility of heat transfer at extreme high temperatures such as $300{ }^{\circ} \mathrm{C}, 350{ }^{\circ} \mathrm{C}, 400{ }^{\circ} \mathrm{C}$ and $450{ }^{\circ} \mathrm{C}$ so that we numerically investigated the possibility of heat transfer at these high-temperature regimes. Actually, the simulation results revealed that even at inflated reservoir temperatures, there is no heat transfer between wellbore and reservoir after the reservoir formation is damaged by fines. Hence, it can be implicated the well will be under that state of adiabatic regardless of the rise in the gas reservoir temperature. Overall, this paper successfully made the numerical demonstration that even at enormous reservoir temperature, the well will behave adiabatic.

\section{References}

Bansal R (2017) Fluid mechanics and hydraulic machines, 9th edn. Laxmi Publications (P) Ltd, New Delhi, pp 540-546

Benjamin G (2012) Heat transfer. Literary Licensing, LLC, Whitefish

Byrne M, Jimenez M, Rojas E, Chavez J (2010) Modelling well inflow potential in three dimensions using computational fluid dynamics. In: SPE international symposium and exhibition on formation damage control. Society of Petroleum Engineers, Lafayette, Louisiana, USA, pp 1-8

Civan F (2010) Non-isothermal permeability impairment by fines migration and deposition in porous media including dispersive transport. In: Transport in porous media, vol 85, no 1, pp 233258, viewed 10 December 2016

Cuevas J, Gasbarri S, Miguel A (2010) Computational fluid dynamics for estimating oil-relative permeability curves. In: SPE Latin American and Caribbean petroleum engineering conference. Society of Petroleum Engineers, Maracaibo, Venezuela, pp 1-34

Dale S (2017) BP energy outlook 2017. BP Plc, London

Dullien F (1992) Porous media: fluid transport and pore structure, 2nd edn. Academic Press, San Diego, pp 35-75

Hagoort J (2005) Prediction of wellbore temperatures in gas production wells. J Pet Sci Eng 49:22-36

Lin R, Shao C, Li J (2013) Study on two-phase flow and heat transfer in offshore wells. J Pet Sci Eng 111:42-49

Mu Y, Mu X (2013) Energy conservation in the Earth's crust and climate change. J Air Waste Manag Assoc 63:150-160

Narasimhan A (2013) Essentials of heat and fluid flow in porous media, 1st edn. CRC Press, Boca Raton. ISBN 978-93-8212-721-5

Raha S, Khilar KC, Kanpur PC (2007) Regularities in pressure filtration of fine and colloidal suspensions. Int J Miner Process 84(1-4):348-360

Ramey HJ Jr (1962) Wellbore heat transmission. J Pet Technol 14:427-435

Randolph JB, Adams B, Kuehn TH, Saar MO (2015) Wellbore heat transfer in $\mathrm{CO}_{2}$-based geothermal systems. University of Minnesota, pp 1-10

Zeinijahromi A, Vaz A, Bedrikovetsky P (2012) Well impairment by fines migration in gas fields. J Pet Sci Eng 88-89:125-135

Zhang J, Yan X, Yang X, Zhao X (2015) Evaluation of wellbore integrity for HTHP gas wells under solid-temperature coupling using a new analytical model. J Nat Sci Eng 25:347-358

Publisher's Note Springer Nature remains neutral with regard to jurisdictional claims in published maps and institutional affiliations. 